\title{
ENTANGLING OSCILLATORS THROUGH ENVIRONMENT NOISE
}

\author{
F. Benatti \\ Dipartimento di Fisica Teorica, Università di Trieste \\ Strada Costiera 11, 34014 Trieste, Italy \\ and \\ Istituto Nazionale di Fisica Nucleare, Sezione di Trieste \\ R. Floreanini \\ Istituto Nazionale di Fisica Nucleare, Sezione di Trieste \\ Dipartimento di Fisica Teorica, Università di Trieste \\ Strada Costiera 11, 34014 Trieste, Italy
}

\begin{abstract}
We consider two independent bosonic oscillators immersed in a common bath, evolving in time with a completely positive, markovian, quasifree (Gaussian) reduced dynamics. We show that an initially separated Gaussian state can become entangled as a result of a purely noisy mechanism. In certain cases, the dissipative dynamics allows the persistence of these bath induced quantum correlations even in the asymptotic equilibrium state.
\end{abstract}




\section{INTRODUCTION}

When two non-interacting, independent systems are immersed in a common bath, decoherence effects are expected to arise counteracting any quantum correlation initially present among the two subsystems [1-5]. In certain circumstances however, the environment can also enhance entanglement. Most commonly, this phenomenon is the result of an hamiltonian coupling between the two subsystems [6-10] generated by the action of the bath; remarkably, it can also occur in the markovian regime through a non-hamiltonian, purely noisy mechanism [11-22].

This environment induced entanglement generation has been identified so far in models involving finite-level systems. In this note we shall instead examine the case of simple infinite dimensional systems following a purely markovian dynamics. More specifically, we shall study the behaviour of two independent bosonic oscillators evolving in time according to a semigroup of completely positive maps. We shall limit our considerations to the phenomenologically relevant set of quasi-free (or Gaussian) states [23-29] and to dynamics that preserve this set, the so-called quasi-free semigroups, whose properties are well studied in the literature [30, 31].

After a brief review of the theory of quasi-free dynamical semigroups in relation to the present situation, we shall first discuss the possibility of entanglement creation at the beginning of the evolution, in the vicinity of the initial time $t=0$. Using the partial transposition criterion for two-mode Gaussian states, we shall derive the condition that assures environment induced entanglement generation as soon as $t>0$.

Although in general entanglement will keep growing also away from the neighborhood of $t=0$, the decoherent, noisy character of the environment will eventually set in. Whether or not quantum correlations are actually left in the asymptotic long time regime is the result of a delicate balance between entanglement generation and decoherence. It is remarkable that for certain environments the long time equilibrium state could indeed retain a nonvanishing entanglement, and an example of this phenomenon is explicitly presented at the end. In view of the direct phenomenological relevance of Gaussian states in quantum optics, we believe that these results might be of help in the design and actual realization of quantum devices and circuits.

\section{GAUSSIAN STATES}

As explained in the introductory remarks, we shall study the dynamics of independent oscillators in weak interaction with a large environment, so that their reduced time evolution can be well represented by a markovian, completely positive dynamics. Although our considerations concerning bath-induced entanglement creation will involve two such oscillators, for sake of generality in this Section and the next will let their number $n$ to be arbitrary. The states of this system will be represented by a density matrix $\rho$, i.e. by a positive hermitian operator, with unit trace, acting on the bosonic Hilbert space $\mathcal{H}$. It can be identified with the tensor product of $n$ independent Fock spaces; they are generated from the vacuum state through the action of polynomials in the creation $a_{i}^{\dagger}$ and annihila- 
tion $a_{i}, i=1,2, \ldots, n$, operators pertaining to the single oscillators. These operators obey the standard bosonic oscillator algebra: $\left[a_{i}, a_{j}^{\dagger}\right]=\delta_{i j},\left[a_{i}^{\dagger}, a_{j}^{\dagger}\right]=0=\left[a_{i}, a_{j}\right]$.

In dealing with this algebra, it is useful to adopt the holomorphic representation [32-35]; it allows working with explicit expressions for both states and operators, including the density matrix $\rho$. In this formulation, the elements $|\psi\rangle$ of the bosonic Hilbert space $\mathcal{H}$ are represented by holomorphic functions $\psi(\bar{z})$ of the set of $n$ complex variables $\bar{z}=$ $\left(\bar{z}_{1}, \bar{z}_{2}, \ldots, \bar{z}_{n}\right)$, with inner product: ${ }^{\dagger}$

$$
\langle\phi \mid \psi\rangle=\int \psi^{*}(z) \phi(\bar{z}) e^{-\sum_{i} \bar{z}_{i} z_{i}} \Pi_{i} \mathrm{~d} \bar{z}_{i} \mathrm{~d} z_{i}
$$

where $*$ signifies complex conjugation. To every operator $\mathcal{O}$ acting on $\mathcal{H}$ there corresponds a kernel $\mathcal{O}(\bar{z}, z)$ of $2 n$ independent complex variables $\bar{z}=\left(\bar{z}_{1}, \bar{z}_{2}, \ldots, \bar{z}_{n}\right)$ and $z=\left(z_{1}, z_{2}, \ldots, z_{n}\right)$, such that for the state $|\phi\rangle=\mathcal{O}|\psi\rangle$ one finds the representation:

$$
\phi(\bar{z})=\int \mathcal{O}(\bar{z}, w) \psi(\bar{w}) e^{-\sum_{i} \bar{w}_{i} w_{i}} \Pi_{i} \mathrm{~d} \bar{w}_{i} \mathrm{~d} w_{i}
$$

In particular, the creation and annihilation operators, when acting on a state $|\psi\rangle$, are realized by multiplication and differentiation by the variables $\bar{z}_{i}$ :

$$
a_{i}^{\dagger}|\psi\rangle \rightarrow \bar{z}_{i} \psi(\bar{z}), \quad a_{i}|\psi\rangle \rightarrow \frac{\partial}{\partial \bar{z}_{i}} \psi(\bar{z})
$$

while the identity operator is represented by $\exp \left(\sum_{i} \bar{z}_{i} z_{i}\right)$.

Among all density matrices $\rho$ for the $n$ oscillators, the so-called quasi-free or Gaussian states are of particular interest: they can be easily produced in experiments in quantum optics [27-29]. In the holomorphic formulation, they can be defined as possessing a representation kernel $\rho(\bar{z}, z)$ of generic Gaussian form:

$$
\rho(\bar{z}, z)=\sqrt{\mathcal{N}} \exp \left[-\frac{1}{2} \mathbf{z}^{T} \cdot \mathbf{G}^{-1} \cdot \mathbf{z}+\sum_{i} \bar{z}_{i} z_{i}\right]
$$

where $\mathbf{z}^{T}$ is a $2 n$-dimensional row vector with components $\left(z_{1}, z_{2}, \ldots, z_{n}, \bar{z}_{1}, \bar{z}_{2}, \ldots, \bar{z}_{n}\right)$, while $\mathbf{z}$ is its corresponding column vector ( $T$ signifies matrix transposition and matrix multiplication). The covariance $\mathbf{G}$ is $2 n \times 2 n$ matrix; with respect to the natural decomposition $\mathbf{z}^{T} \equiv(z \bar{z})$ into the set of variables $z_{i}$ and $\bar{z}_{i}$, it can be conveniently parametrized as

$$
\mathbf{G}=\left(\begin{array}{cc}
\hat{\alpha} & \hat{\beta} \\
\hat{\beta}^{T} & \hat{\alpha}^{*}
\end{array}\right)
$$

where $\hat{\alpha}$ and $\hat{\beta}$ are $n \times n$ matrices, with $\hat{\alpha}$ symmetric and $\hat{\beta}$ hermitian to assure the hermiticity of $\rho$. They represent the averages in the given state $\rho$ of quadratic operators

\footnotetext{
$\dagger$ Here and in the following we use the conventions of Ref.[32]
} 
in the creation and annihilation operators:

$$
\begin{aligned}
\left\langle a_{i} a_{j}\right\rangle & \equiv \operatorname{Tr}\left[a_{i} a_{j} \rho\right]=\int \frac{\partial^{2}}{\partial \bar{z}_{i} \partial \bar{z}_{j}}[\rho(\bar{z}, z)] \mathrm{e}^{-\sum_{k} \bar{z}_{k} z_{k}} \Pi_{k} \mathrm{~d} \bar{z}_{k} \mathrm{~d} z_{k}=\hat{\alpha}_{i j} \\
\left\langle a_{i}^{\dagger} a_{j}^{\dagger}\right\rangle & \equiv \operatorname{Tr}\left[a_{i}^{\dagger} a_{j}^{\dagger} \rho\right]=\int \bar{z}_{j} \bar{z}_{j} \rho(\bar{z}, z) \mathrm{e}^{-\sum_{k} \bar{z}_{k} z_{k}} \Pi_{k} \mathrm{~d} \bar{z}_{k} \mathrm{~d} z_{k}=\hat{\alpha}_{i j}^{*} \\
\left\langle a_{i} a_{j}^{\dagger}\right\rangle & \equiv \operatorname{Tr}\left[a_{i} a_{j}^{\dagger} \rho\right]=\int \frac{\partial}{\partial \bar{z}_{i}}\left[\bar{z}_{j} \rho(\bar{z}, z)\right] \mathrm{e}^{-\sum_{k} \bar{z}_{k} z_{k}} \Pi_{k} \mathrm{~d} \bar{z}_{k} \mathrm{~d} z_{k}=\hat{\beta}_{i j}
\end{aligned}
$$

As a result of the last relation, the matrix $\hat{\beta}$ turns out to be non-negative. For simplicity, in writing (2.4) we have assumed $\left\langle a_{i}^{\dagger}\right\rangle=\left\langle a_{i}\right\rangle=0$; this condition can be easily released starting with a more general Ansatz for $\rho(\bar{z}, z)$, containing in the exponential also linear terms in $z_{i}$ and $\bar{z}_{i}$; it will not be needed for the considerations that follow. The trace condition,

$$
\operatorname{Tr}[\rho]=\int \rho(\bar{z}, z) \mathrm{e}^{-\sum_{i} \bar{z}_{i} z_{i}} \Pi_{i} \mathrm{~d} \bar{z}_{i} \mathrm{~d} z_{i}=1,
$$

further fixes the normalization constant, $\mathcal{N}=\operatorname{det}(\mathbf{G})$, provided the previous integral makes sense [32]. Indeed, convergence of (2.7), as well as of the integrals in (2.6), put further constraints on the entries of the covariance matrix $\mathbf{G}$, or equivalently on those of $\hat{\alpha}$ and $\hat{\beta}$. As similarly done with the vector $\mathbf{z}$, let us collect the $2 n$ annihilation and creation operators $a_{i}$ and $a_{i}^{\dagger}$ into the column vector a and its hermitan conjugate row vector $\mathbf{a}^{\dagger} \equiv\left(a_{i}^{\dagger} a_{i}\right)=\left(a_{1}^{\dagger}, a_{2}^{\dagger}, \ldots, a_{n}^{\dagger}, a_{1}, a_{2}, \ldots, a_{n}\right)$. Then, necessarily, the following $2 n \times 2 n$ matrix of bilinear expectation values results non negative:

$$
\left\langle\mathbf{a}_{\mu} \mathbf{a}_{\nu}^{\dagger}\right\rangle \equiv \operatorname{Tr}\left[\mathbf{a}_{\mu} \mathbf{a}_{\nu}^{\dagger} \rho\right] \geq 0, \quad \mu, \nu=1,2, \ldots, 2 n .
$$

It turns out that (2.8) is also a sufficient condition for the expression in (2.4) to represent a physical state [23, 36-38].

It is customary to rewrite this condition in terms of the $2 n \times 2 n$ matrix $\mathbf{V}$ of symmetric bilinears:

$$
\mathbf{V}_{\mu \nu}=\frac{1}{2}\left\langle\left\{\mathbf{a}_{\mu}, \mathbf{a}_{\nu}^{\dagger}\right\}\right\rangle \equiv \frac{1}{2} \operatorname{Tr}\left[\left(\mathbf{a}_{\mu} \mathbf{a}_{\nu}^{\dagger}+\mathbf{a}_{\nu}^{\dagger} \mathbf{a}_{\mu}\right) \rho\right]
$$

in terms of the natural decomposition of $\mathbf{a}^{\dagger} \equiv\left(a_{i}^{\dagger} a_{i}\right)$ into the set of all creation and annihilation operators, it can be explicitly written as:

$$
\mathbf{V}=\left(\begin{array}{cc}
\hat{\beta} & \hat{\alpha} \\
\hat{\alpha}^{*} & \hat{\beta}^{T}
\end{array}\right)-\frac{\mathbf{1}}{2} .
$$

Introducing also the $2 n \times 2 n$ matrix of commutators

$$
\boldsymbol{\Sigma}_{\mu \nu} \equiv\left\langle\left[\mathbf{a}_{\mu}, \mathbf{a}_{\nu}^{\dagger}\right]\right\rangle=\left(\begin{array}{cc}
1 & 0 \\
0 & -1
\end{array}\right)
$$

one can finally express the positivity condition (2.8) as:

$$
\mathbf{V}+\frac{\boldsymbol{\Sigma}}{2} \geq 0
$$


In the following, we shall limit our considerations to the set of Gaussian states, i.e. to the states $\rho$ represented by kernels of the form (2.4) satisfying the condition (2.8), or equivalently (2.12).

A particularly important class of Gaussian states are the pure ones. In the holomorphic representation they are described by properly normalized Gaussian functions of the variables $\bar{z}_{i}$ :

$$
\Psi_{\Omega}(\bar{z})=\operatorname{det}^{1 / 4}\left(1-\Omega^{*} \Omega\right) \exp \left[-\frac{1}{2} \sum_{i, j=1}^{n} \bar{z}_{i} \Omega_{i j} \bar{z}_{j}\right],
$$

with $\Omega$ a complex, symmetric matrix, such that $|\Omega| \leq 1$ to guarantee norm convergence. The corresponding kernel $\rho(\bar{z}, z) \equiv \Psi_{\Omega}(\bar{z}) \Psi_{\Omega}^{*}(z)$ can be cast in the form $(2.4)$, with submatrix coefficients $\hat{\alpha}=-\Omega\left(1-\Omega^{*} \Omega\right)^{-1}$ and $\hat{\beta}=\left(1-\Omega^{*} \Omega\right)^{-1}$, respectively.

\section{QUASI-FREE QUANTUM DYNAMICAL SEMIGROUPS}

Our analysis is based on the assumption that the time evolution of the set of independent oscillators immersed in the common bath be markovian and given by a quantum dynamical semigroup; this is a completely positive, trace preserving, one parameter family of linear maps, acting on the set of density matrices $\rho$ representing the oscillator states. These maps are generated by equations of the following Kossakowski-Lindblad form [39-41]:

$$
\frac{\partial \rho(t)}{\partial t}=\mathcal{L}[\rho(t)] \equiv-i[H, \rho(t)]+L[\rho(t)]
$$

with $H$ and effective hamiltonian and $L$ a dissipative piece, that can be abstractly written as

$$
L[\rho]=\sum_{k}\left(L_{k} \rho L_{k}^{\dagger}-\frac{1}{2}\left\{L_{k}^{\dagger} L_{k}, \rho\right\}\right)
$$

in terms of a collection of suitable, well-behaved operators $L_{k}$.

Being interested in the set of Gaussian states, we would like to characterize those quantum dynamical semigroups that preserve that set. Although already discussed using abstract, mathematically rigorous techniques [30,31], this problem has a simple and direct solution in the holomorphic representation: the semigroup generated by (3.1) leaves the form (2.4) for $\rho$ invariant provided $L_{k}$ is a linear and $H$ a quadratic combination of the creation and annihilation operators. Indeed, only in this case the r.h.s. of (3.1) results quadratic in the variables $\bar{z}_{i}$ and their derivatives when acting on the representation kernel $\rho(\bar{z}, z)$, thus preserving its generic form (2.4); as a consequence, the evolution equation (3.1) reduces to a linear, differential equation for the entries $\hat{\alpha}$ and $\hat{\beta}$ of the covariance matrix $\mathbf{G}$ (given explicitly in (3.7) below).

In view of this result, the effective hamiltonian will be taken to have the generic quadratic form

$$
H=\frac{1}{2} \sum_{i, j=1}^{n} \hat{\omega}_{i j}\left\{a_{i}^{\dagger}, a_{j}\right\}
$$


whith the matrix $\hat{\omega}$ hermitian and positive. Note that terms containing $a_{i} a_{j}$ and their hermitian conjugate can be eliminated by a suitable unitary canonical transformation $[32,34,35]$, and therefore do not appear in (3.2). Similarly, the most general allowed dissipative term takes the following form:

$$
\begin{aligned}
L[\rho]=\frac{1}{2} \sum_{i, j=1}^{n}\{ & \left\{\hat{\eta}_{i j}\left(\left[a_{j} \rho, a_{i}^{\dagger}\right]+\left[a_{j}, \rho a_{i}^{\dagger}\right]\right)+\hat{\sigma}_{i j}\left(\left[a_{j}^{\dagger} \rho, a_{i}\right]+\left[a_{j}^{\dagger}, \rho a_{i}\right]\right)\right. \\
& \left.+\hat{\lambda}_{i j}\left(\left[a_{j} \rho, a_{i}\right]+\left[a_{j}, \rho a_{i}\right]\right)+\hat{\lambda}_{j i}^{*}\left(\left[a_{j}^{\dagger} \rho, a_{i}^{\dagger}\right]+\left[a_{j}^{\dagger}, \rho a_{i}^{\dagger}\right]\right)\right\}
\end{aligned}
$$

where the $n \times n$ coefficients matrices $\hat{\eta}, \hat{\sigma}$ and $\hat{\lambda}$ encode the physical properties of the environment and can be expressed in terms of the Fourier transform of the correlation functions in the bath [1-5].

These parameters are not completely arbitrary. First of all, $\hat{\eta}$ and $\hat{\sigma}$ need to be hermitian in order to comply with the hermiticity preserving requirement of the generated semigroup. In addition, the request of complete positivity gives rise to further constraints. Indeed, by using the $2 n$-dimensional vectors $\mathbf{a}$ and $\mathbf{a}^{\dagger}$ introduced in the previous section, the dissipative term in (3.4) can be recast in compact form as:

$$
L[\rho]=\sum_{\mu, \nu=1}^{2 n} \mathbf{C}_{\mu \nu}\left(\mathbf{a}_{\nu} \rho \mathbf{a}_{\mu}^{\dagger}-\frac{1}{2}\left\{\mathbf{a}_{\mu}^{\dagger} \mathbf{a}_{\nu}, \rho\right\}\right)
$$

where the bath coefficients are now embedded in the $2 n \times 2 n$ Kossakowski matrix $\mathbf{C}$. It is well known that the requirement of complete positivity of the dynamics generated by a dissipative term $L[\rho]$ in the form (3.5) is equivalent to the positivity of the Kossakowski matrix [39-41]; in the present case, using the block decomposition introduced before in writing (2.10), this condition explicitly reads:

$$
\mathbf{C}=\left(\begin{array}{cc}
\hat{\eta} & \hat{\lambda}^{\dagger} \\
\hat{\lambda} & \hat{\sigma}
\end{array}\right) \geq 0
$$

The finite-time evolution maps obtained from the equation (3.1), with hamiltonian as in (3.3) and dissipative term as in (3.5), with $\mathbf{C} \geq 0$, are known in the literature as quasifree quantum dynamical semigroups [30, 31]: as already observed, they are characterized by the property of transforming the set of quasi-free (Gaussian) states into itself.

Inserting the general Ansatz (2.4) for the kernel $\rho(\bar{z}, z ; t)$ in the evolution equation (3.1), with (3.3) and (3.4), and using the prescriptions (2.3), one easily derives the equation obeyed by the time-dependent covariance $\mathbf{G}(t)$, or equivalently those for the submatrices $\hat{\alpha}$ and $\hat{\beta}$. It can be more conveniently rewritten as an equation for the symmetric covariance matrix $\mathbf{V}$ introduced in (2.9):

$$
\partial_{t} \mathbf{V}(t)=\mathbf{A}^{\dagger} \cdot \mathbf{V}(t)+\mathbf{V}(t) \cdot \mathbf{A}+\mathbf{B}
$$


The $2 n \times 2 n$ matrices $\mathbf{A}$ and $\mathbf{B}$ contain the dependence on the hamiltonian $\hat{\omega}_{i j}$ and dissipative coefficients $\hat{\eta}_{i j}, \hat{\sigma}_{i j}, \hat{\lambda}_{i j}$; using the same decomposition introduced in the definition of $\mathbf{V}$ in (2.9), one explicitly finds:

$$
\mathbf{A}=\frac{1}{2}\left(\begin{array}{cc}
\hat{\sigma}^{*}-\hat{\eta}+2 i \hat{\omega} & -2\left(\hat{\lambda}^{(A)}\right)^{*} \\
-2 \hat{\lambda}^{(A)} & \hat{\sigma}-\hat{\eta}^{*}-2 i \hat{\omega}^{*}
\end{array}\right) \quad \mathbf{B}=\frac{1}{2}\left(\begin{array}{cc}
\hat{\sigma}^{*}+\hat{\eta} & -2\left(\hat{\lambda}^{(S)}\right)^{*} \\
-2 \hat{\lambda}^{(S)} & \hat{\sigma}+\hat{\eta}^{*}
\end{array}\right),
$$

where $\hat{\lambda}_{i j}^{(S)}$ and $\hat{\lambda}_{i j}^{(A)}$ are respectively the symmetric and antisymmetric parts of the complex matrix $\hat{\lambda}_{i j}$.

The evolution equation (3.7) has been originally derived using the equivalent Heisenberg picture where the time-evolution affects the observables $\mathcal{O}$ instead of the states $\rho$; the two pictures are connected by the duality relation involving expectation values

$$
\langle\mathcal{O}\rangle(t)=\operatorname{Tr}\left[\gamma_{t}(\rho) \mathcal{O}\right]=\operatorname{Tr}\left[\rho \Gamma_{t}(\mathcal{O})\right] ;
$$

the semigroup $\gamma_{t}$ is generated by (3.1), while $\Gamma_{t}$ by the dual equation:

$$
\frac{\partial \mathcal{O}(t)}{\partial t}=i[H, \mathcal{O}(t)]+\sum_{\mu, \nu=1}^{2 n} \mathbf{C}_{\mu \nu}\left(\mathbf{a}_{\mu}^{\dagger} \mathcal{O}(t) \mathbf{a}_{\nu}-\frac{1}{2}\left\{\mathbf{a}_{\mu}^{\dagger} \mathbf{a}_{\nu}, \mathcal{O}(t)\right\}\right) .
$$

Recalling the definition (2.9) and inserting in place of $\mathcal{O}$ suitable bilinears in the creation and annihilation operators, one sees that indeed the above equation readily implies (3.7).

Being a first order differential equation involving finite-dimensional matrices, the evolution equation (3.7) can be easily integrated. Its solution involves the exponentiation of A and explicitly reads:

$$
\mathbf{V}(t)=\mathrm{e}^{t \mathbf{A}^{\dagger}} \cdot \mathbf{V}(0) \cdot \mathrm{e}^{t \mathbf{A}}+\int_{0}^{t} \mathrm{~d} \tau \mathrm{e}^{\tau \mathbf{A}^{\dagger}} \cdot \mathbf{B} \cdot \mathrm{e}^{\tau \mathbf{A}} .
$$

In the next Sections, the dynamical evolution given by (3.11) will be used to investigate whether and under what conditions it can give rise to entanglement enhancement.

\section{ENVIRONMENT INDUCED ENTANGLEMENT GENERATION}

In order to study the possibility of entanglement generation by the external bath, it sufficies to consider a system formed by two oscillators. Henceforth, we specialize $n=2$, so that the covariance $\mathbf{V}$, as well as the Kossakowski $\mathbf{C}$ and the coefficients $\mathbf{A}, \mathbf{B}$, all become $4 \times 4$ matrices, and correspondingly $\hat{\eta}, \hat{\sigma}$ and $\hat{\lambda}$ result $2 \times 2$ matrices. We shall concentrate our attention on discussing bath assisted entanglement production by purely dissipative mechanism; we shall therefore ignore any hamiltonian coupling between the two oscillators, setting in particular $\hat{\omega}_{i j}=0 .^{\dagger}$

$\dagger$ The entanglement power of purely hamiltonian couplings have been extensively studied in the literature, e.g. see [6-10]. 
Let us first analyze the possibility of entanglement creation at the beginning of the evolution, in the neighborhood of $t=0$. As initial state of the two-oscillator system we shall choose a kernel $\rho(\bar{z}, z ; 0)$ representing a separable Gaussian state and use the partial transposition criterion $[42,43]$ to check whether $\rho(\bar{z}, z ; t)$ becomes entangled at a later time $t$.

As well known, in quantum mechanics the operation of full transposition on any state $\rho$ corresponds to the time-reversal transformation: it can be easily implemented in the holomorphic representation through the variable exchange $\bar{z}_{i} \leftrightarrow z_{i}$ in the kernel $\rho(\bar{z}, z)$. Recalling the expression in (2.4), this exchange equivalently corresponds to the transformations $\hat{\alpha} \leftrightarrow \hat{\alpha}^{*}$ and $\hat{\beta} \leftrightarrow \hat{\beta}^{T}$ on the submatrices defining the covariance $\mathbf{G}$, and thus to the exchange $a_{i} \leftrightarrow a_{i}^{\dagger}$ in the definition (2.9) of symmetric covariance $\mathbf{V}$. Clearly, the transformed $\mathbf{V}$ still satisfies the positivity condition (2.12) if the original covariance does, so that full transposition maps the set of Gaussian density matrices into itself.

This is not the case for the operation of partial transposition, i.e. of transposition involving only one of the two oscillator system, say the first one, so that $\bar{z}_{1} \leftrightarrow z_{1}$. It clearly maps states into states for separable ones, but not in general for correlated ones: it thus provides a sufficient criterion for bipartite entanglement in any dimensions.

For the case at hand, one finds the the operation of partial transposition with respect to the first system results in the following transformation of the $4 \times 4$ symmetric covariance:

$$
\mathbf{V} \rightarrow \widetilde{\mathbf{V}}=\mathbf{T} \cdot \mathbf{V} \cdot \mathbf{T}, \quad \mathbf{T}=\left(\begin{array}{llll}
0 & 0 & 1 & 0 \\
0 & 1 & 0 & 0 \\
1 & 0 & 0 & 0 \\
0 & 0 & 0 & 1
\end{array}\right)
$$

We have seen that in order for a Gaussian kernel $\rho(\bar{z}, z)$ in $(2.4)$ to represent a state, the condition (2.12) on its corresponding symmetric covariance $\mathbf{V}$ needs to be satisfied; if one further finds:

$$
\widetilde{\mathbf{V}}+\frac{\boldsymbol{\Sigma}}{2}<0
$$

then the state is surely entangled. In the two-mode case we are studying, also the converse is true, namely, if $\mathbf{V}$ represents the symmetric covariance of an entangled Gaussian state, then (4.2) is necessarily satisfied [44].

In the case of the quasi-free markovian dynamics in (3.11), instead of dealing directly with the behaviour in time of $\widetilde{\mathbf{V}}(t)$ and the inequality (4.2), we find it more convenient to consider the scalar quantity:

$$
\mathcal{Q}(t)=\left\langle\Psi\left|\left(\widetilde{\mathbf{V}}(t)+\frac{\boldsymbol{\Sigma}}{2}\right)\right| \Psi\right\rangle,
$$

where $|\Psi\rangle$ is a four-dimensional complex vector, choosen in the null eigenspace of the matrix $\tilde{\mathbf{V}}(0)+\boldsymbol{\Sigma} / 2$, so that $\mathcal{Q}(0)=0$. Then, the two oscillators, initially prepared in a separable Gaussian state, will start to become correlated by the noisy dynamics induced by the bath in which they are immersed if a suitable vector $|\Psi\rangle$ exists such that:

$$
\partial_{t} \mathcal{Q}(0)<0
$$


By applying the partial transposition operation $\mathbf{T}$ to both sides of (3.7) and defining:

$$
|\widetilde{\Psi}\rangle=\mathbf{T}|\Psi\rangle, \quad \widetilde{\boldsymbol{\Sigma}}=\mathbf{T} \cdot \boldsymbol{\Sigma} \cdot \mathbf{T}=\left(\begin{array}{cc}
-\sigma_{3} & 0 \\
0 & \sigma_{3}
\end{array}\right)
$$

with $\sigma_{3}$ the third Pauli matrix, the condition (4.4) can be cast in the following form:

$$
2\langle\widetilde{\Psi}|\mathbf{B}| \widetilde{\Psi}\rangle<\left\langle\widetilde{\Psi}\left|\left[\mathbf{A}^{\dagger} \cdot \widetilde{\boldsymbol{\Sigma}}+\widetilde{\boldsymbol{\Sigma}} \cdot \mathbf{A}\right]\right| \widetilde{\Psi}\right\rangle,
$$

explicitly showing the dependence on the bath through the presence of the coefficients matrices $\mathbf{A}$ and $\mathbf{B}$.

As initial state for the two oscillators we take a separable state that is also pure; this is by no means a restriction: if the bath is not able to entangle pure states, it will surely not correlate their mixtures. Gaussian pure states are represented by holomorphic functions of the form (2.13), where now the matrix $\Omega$ is two-dimensional. Separability further impose the vanishing of the off-diagonal elements, so that $\Omega=\operatorname{diag}\left(\Omega_{1}, \Omega_{2}\right)$, with $\left|\Omega_{i}\right| \leq 1$ to assure a finite state norm. Similarly, also the $2 \times 2$ submatrix $\hat{\alpha}$ and $\hat{\beta}$, defining the covariances $\mathbf{G}$ and $\mathbf{V}$, turn out to be diagonal:

$$
\hat{\alpha}=\left(\begin{array}{cc}
\frac{\Omega_{1}}{1-\left|\Omega_{1}\right|^{2}} & 0 \\
0 & \frac{\Omega_{2}}{1-\left|\Omega_{2}\right|^{2}}
\end{array}\right), \quad \hat{\beta}=\left(\begin{array}{cc}
\frac{1}{1-\left|\Omega_{1}\right|^{2}} & 0 \\
0 & \frac{1}{1-\left|\Omega_{2}\right|^{2}}
\end{array}\right) .
$$

The condition (2.12),

$$
\mathbf{V}+\frac{\boldsymbol{\Sigma}}{2}=\left(\begin{array}{cc}
\hat{\beta} & \hat{\alpha} \\
\hat{\alpha}^{*} & \hat{\beta}^{T}-1
\end{array}\right) \geq 0,
$$

that assures the positivity of the corresponding Gaussian state, gives again the constraints $\left|\Omega_{i}\right| \leq 1$, as it should, since (4.8) is equivalent to norm convergence.

The null subspace of the combination $\mathbf{V}+\boldsymbol{\Sigma} / 2$ in (4.8) results two-dimensional; it is spanned by the vector $|\Psi\rangle$ of components $\left(a \Omega_{1}^{*}, b \Omega_{2}, a, b\right)$, with $a$ and $b$ abitrary complex parameters. Then, entanglement between the two oscillators will surely occur in baths for which (4.6) is satisfied with this choice of $|\Psi\rangle$.

To show that indeed this is possible, let us initially prepare the two oscillators in their corresponding zero-temperature Fock vacua, so that $\Omega_{1}=\Omega_{2}=0$. By further choosing the parameters $a$ and $b$ real and equal, one finds that the inequality (4.6) reduces to:

$$
\hat{\sigma}_{11}+\hat{\sigma}_{22}<\mathcal{R} e\left(\hat{\lambda}_{12}+\hat{\lambda}_{21}\right),
$$

involving the entries of the $2 \times 2$ matrices $\hat{\sigma}$ and $\hat{\lambda}$ that appear in the Kossakowski matrix $\mathbf{C}$ in (3.6). As discussed in Section 3, the matrix $\mathbf{C}$ parametrizes the physical properties of the bath: it needs to be positive in order to comply with the requirement of complete positivity of the reduced dynamics of the two oscillators. Therefore, in order to be sure that baths satisfying (4.9) can actually be constructed, one needs to check the compatibility of (4.9) with the condition of positivity of the Kossakowski matrix. To explicitly show this, it is enough to take the matrices $\hat{\eta}$ and $\hat{\sigma}$ diagonal and $\hat{\lambda}$ real, with only the entry $\hat{\lambda}_{21}$ non 
vanishing. Then, the condition $\mathbf{C} \geq 0$ simply gives: $\hat{\eta}_{11} \geq 0, \hat{\eta}_{22} \geq 0, \hat{\sigma}_{11} \geq 0, \hat{\sigma}_{22} \geq 0$ and $\hat{\lambda}_{21}^{2} \leq \hat{\eta}_{22} \hat{\sigma}_{11}$. Combining these conditions with that in (4.9), one obtains (for $\hat{\sigma}_{11} \neq 0$ ):

$$
1+\frac{\hat{\sigma}_{22}}{\hat{\sigma}_{11}}<\frac{\hat{\lambda}_{21}}{\hat{\sigma}_{11}} \leq\left(\frac{\hat{\eta}_{22}}{\hat{\sigma}_{11}}\right)^{1 / 2} .
$$

As a consequence, in order to be able to correlate the two oscillators, originally prepared in the separated Fock vacuum, it is sufficient to immerse them in a bath for which $\lambda_{21}$ is as in (4.10) and $\hat{\eta}_{22}$ greater than $\left(\hat{\sigma}_{11}+\hat{\sigma}_{22}\right)^{2} / \hat{\sigma}_{11}$.

Environments that are able to entangle two oscillators that are prepared in separable temperature states can similarly be found. They are characterized by a Kossakowski matrix of the form:

$$
\mathbf{C}=\left(\begin{array}{cc}
\eta & \lambda^{*} \\
\lambda & \sigma
\end{array}\right) \otimes\left(\begin{array}{ll}
1 & 1 \\
1 & 1
\end{array}\right)
$$

where the parameters $\eta$ and $\sigma$ are real and positive, while $\lambda$ is complex, satisfying the positivity condition: $|\lambda|^{2} \leq \eta \sigma$. The initial state covariance is characterized by submatrices $\hat{\alpha}$ and $\hat{\beta}$ as in (4.7); for simplicity, let us assume the single oscillator states to be equal, so that $\Omega_{1}=\Omega_{2}=\Omega$. By taking the norm of the parameters $a$ and $b$ appearing in the vector $|\Psi\rangle$ to satisfy $|a|=|b|=1 / \sqrt{2}$, and further adjusting their phases and that of $\lambda$ according to the relation $\operatorname{Arg}(\lambda)=\operatorname{Arg}(a)-\operatorname{Arg}(b)=\pi-\operatorname{Arg}(\Omega)$, one checks that the condition (4.6) assuring entanglement generation can be fulfilled by choosing

$$
|\lambda|>\frac{\sigma-|\Omega| \eta}{1-|\Omega|}
$$

which is compatible with the positivity condition of the above Kossakowski matrix provided we further take $\eta>\sigma$.

\section{ASYMPTOTIC ENTANGLEMENT}

In the previous Section we have discussed examples of baths capable of correlate two independent oscillators through a purely noisy mechanism: no direct hamiltonian couplings between the two subsystems were present. This happens at the beginning of the evolution, as soon as $t>0$. The test (4.4), on which this conclusion is based, is however unable to determine the fate of this entanglement as time becomes large.

On general grounds, one expects that the effects of decoherence, counteracting entanglement production, be dominant in the large time region, so that no quantum correlation is expected to be left at infinity. Nevertheless, there are situations in which entanglement is found to keep growing, reaching at the end an asymptotic non-vanishing value. An example is provided by baths for which the corresponding Kossakowski matrix takes the form in (4.11). 
In this case, the evolution equation (3.1) can be rewritten in a simplified form by introducing the following set of independent oscillator variables:

$$
\begin{array}{ll}
A=\frac{a_{1}+a_{2}}{\sqrt{2}} & A^{\dagger}=\frac{a_{1}^{\dagger}+a_{2}^{\dagger}}{\sqrt{2}} \\
B=\frac{a_{1}-a_{2}}{\sqrt{2}} & B^{\dagger}=\frac{a_{1}^{\dagger}-a_{2}^{\dagger}}{\sqrt{2}},
\end{array}
$$

so that: $\left[A, A^{\dagger}\right]=\left[B, B^{\dagger}\right]=1$ and all other commutators vanish. Then, taking into account (4.11), one finds that the equation (3.1) only involves the operators $A$ and $A^{\dagger}$ :

$$
\frac{\partial \rho}{\partial t}=-i \omega\left[A^{\dagger} A, \rho\right]+L[\rho(t)]
$$

where,

$$
\begin{aligned}
L[\rho]=\eta\left(\left[A \rho, A^{\dagger}\right]+\left[A, \rho A^{\dagger}\right]\right) & +\sigma\left(\left[A^{\dagger} \rho, A\right]+\left[A^{\dagger}, \rho A\right]\right) \\
& -\lambda[A,[A, \rho]]-\lambda^{*}\left[A^{\dagger},\left[A^{\dagger}, \rho\right]\right] .
\end{aligned}
$$

For sake of generality, we have also included an hamiltonian contribution; it comes from an effective Hamiltonian of the form (3.3), where, in analogy to (4.11), the $2 \times 2$ matrix $\hat{\omega}$ is taken to be:

$$
\hat{\omega}_{i j}=\omega\left(\begin{array}{ll}
1 & 1 \\
1 & 1
\end{array}\right), \quad \omega>0 .
$$

The general solution of the now single-oscillator dissipative evolution (5.2), (5.3), can be espressed in Gaussian form, using the holomorphic representation involving just one complex variable: $A^{\dagger} \rightarrow \bar{z}, A \rightarrow \partial / \partial \bar{z}$. It can be explicitly expressed in terms of the analogs of the bilinears introduced in (2.6), now just numbers, $\alpha \equiv\left\langle A^{2}\right\rangle$ and $\beta \equiv\left\langle A A^{\dagger}\right\rangle$, that parametrize the covariance of the corresponding Gaussian kernel solution $\rho(\bar{z}, z ; t)$. Notice that we have $\langle A\rangle=\left\langle A^{\dagger}\right\rangle=0$ as a consequence of previous choice $\left\langle a_{i}\right\rangle=\left\langle a_{i}^{\dagger}\right\rangle=0$. One finds that the general solution of the evolution equations for $\alpha(t)$ and $\beta(t)$ derived from (5.2) is given by [45]:

$$
\begin{aligned}
& \alpha(t)=e^{-2(\eta-\sigma+i \omega) t}\left(\alpha_{0}-\alpha_{\infty}\right)+\alpha_{\infty}, \\
& \beta(t)=e^{-2(\eta-\sigma) t}\left(\beta_{0}-\beta_{\infty}\right)+\beta_{\infty},
\end{aligned}
$$

where the parameters $\alpha_{0} \equiv \alpha(0)$ and $\beta_{0} \equiv \beta(0)$ identify the initial values, while

$$
\alpha_{\infty}=\frac{\lambda^{*}(\sigma-\eta+i \omega)}{(\eta-\sigma)^{2}+\omega^{2}}, \quad \beta_{\infty}=\frac{\eta}{\eta-\sigma} .
$$

An asymptotic equilibrium state exists only when $\eta>\sigma$, condition that it will henceforth assumed. Then, the corresponding asymptotic Gaussian kernel is characterized by a covariance with parameters $\alpha$ and $\beta$ as in (5.6); it is not a thermal state, unless $\lambda=0$, in which case the temperature $T$ is given by $\exp (\omega / T)=\eta / \sigma .^{\dagger}$

$\dagger$ Note that this result does not contradict the previous conclusions concerning bathinduced entanglement creation, that in fact requires $\lambda \neq 0$. 
On the other hand, all averages involving $B$ and $B^{\dagger}$ result time independent, since (5.2) does not contain any dependence from these operators; for simplicity, we shall take them to be all zero, except for the bilinear $\left\langle B B^{\dagger}\right\rangle$ : for reasons that it will appear clear soon, it is assumed to be equal to $\beta_{\infty}$, the long-time asymptotic value of $\left\langle A A^{\dagger}\right\rangle$.

Applying back the linear transformations (5.1), these results can now be used to study the evolution of the true physical oscillators, those described by canonical operators $a_{i}$ and $a_{i}^{\dagger}$. As a result, the two-oscillator state is represented by a time dependent Gaussian kernel $\rho(\bar{z}, z ; t)$ giving rise to a covariance matrix $\mathbf{V}(t)$ as in $(2.10)$ :

$$
\mathbf{V}(t)=\left(\begin{array}{cc}
\hat{\beta}(t) & \hat{\alpha}(t) \\
\hat{\alpha}^{*}(t) & \hat{\beta}^{T}(t)
\end{array}\right)-\frac{\mathbf{1}}{2} .
$$

Its time dependence is encoded into the two functions defined in (5.5), that appear in the entries of its two $2 \times 2$ submatrices $\hat{\alpha}_{i j}$ and $\hat{\beta}_{i j}$; explicitly, one finds:

$$
\hat{\alpha}_{i j}(t)=\frac{\alpha(t)}{2}\left(\begin{array}{ll}
1 & 1 \\
1 & 1
\end{array}\right) \quad \hat{\beta}_{i j}(t)=\frac{1}{2}\left(\begin{array}{cc}
\beta(t)+\beta_{\infty} & \beta(t)-\beta_{\infty} \\
\beta(t)-\beta_{\infty} & \beta(t)+\beta_{\infty}
\end{array}\right) .
$$

For sake of simplicity, we shall further take $\alpha(0) \equiv \alpha_{0}=0$, so that $\mathbf{V}(0)$ only involves $\beta_{0}$ and $\beta_{\infty}$. The parameter $\beta_{0}$ is not completely arbitrary: the positivity condition (2.12), $\mathbf{V}(0)+\boldsymbol{\Sigma} / 2 \geq 0$, readily implies $\beta_{0} \geq 1$. Note that this two-mode initial state is mixed (compare it with the pure case in (4.7)) and further separable; indeed, after applying the partial transposition operation (4.1), one finds that

$$
\widetilde{\mathbf{V}}(0)+\frac{\boldsymbol{\Sigma}}{2} \geq 0 ;
$$

indeed, this condition is equivalent to the inequality $2 \beta_{0} \beta_{\infty} \geq \beta_{0}+\beta_{\infty}$, always satisfied for $\beta_{0}, \beta_{\infty} \geq 1$.

We have previously shown that an environment described by a Kossakowski matrix in the form (4.11) is able to initially entangle two independent oscillators immersed in it. To see whether this still holds in the asymptotic long-time regime, one needs to examine the properties of the covariance $\mathbf{V}_{\infty}$ of the corresponding equilibrium state $\rho_{\infty}(\bar{z}, z)$, obtained by letting $\alpha(t) \rightarrow \alpha_{\infty}, \beta(t) \rightarrow \beta_{\infty}$ in the expressions (5.7), (5.8). By using the partial trace criterion, $\rho_{\infty}$ will be entangled if and only if the $4 \times 4$ matrix $\widetilde{\mathbf{V}}_{\infty}+\boldsymbol{\Sigma} / 2$ posseses negative eigenvalues. In the present case, these eigenvalues can be analytically evaluated to be $\beta_{\infty} \pm \sqrt{\Delta_{ \pm}} / 2-1 / 2, \Delta_{ \pm}=1+2\left|\alpha_{\infty}\right|^{2} \pm\left|\alpha_{\infty}\right|\left(1+\left|\alpha_{\infty}\right|^{2}\right)^{1 / 2}$, for all four possible combinations of the \pm signs. Recalling the definitions (5.6), one easily sees that the lowest eigenvalue $\beta_{\infty}-\sqrt{\Delta_{+}} / 2-1 / 2$ can always be made negative by a suitable choice of the bath parameter $\lambda^{\dagger}$

This result is remarkable: it shows that a dissipative quasi-free dynamics, generated by an equation of the form (3.1), (3.4), can produce quantum correlations even for large

$\dagger$ The actual condition reads: $|\lambda|^{2}>4 \eta^{2} \sigma^{2}\left[(\eta-\sigma)^{2}+\omega^{2}\right] /\left(\eta^{2}-\sigma^{2}\right)^{2}$, which is compatible with the requirement of complete positivity, $|\lambda|^{2} \leq \eta \sigma$, for sufficiently small $\omega$. 
times, allowing at the end an entangled equilibrium state. In view of the increasing interest that the theory of quantum information with continous variables is presently attracting, these results may be relevant both in phenomenological and experimental applications. In particular, the posssibility of mantaining bipartite entanglement in a noisy environment even for asymptotic long times may help the actual realizations of simple quantum devices in quantum optics and condensed matter physics.

\section{REFERENCES}

1. R. Alicki and K. Lendi, Quantum Dynamical Semigroups and Applications, Lect. Notes Phys. 286, (Springer-Verlag, Berlin, 1987)

2. V. Gorini, A. Frigerio, M. Verri, A. Kossakowski and E.C.G. Surdarshan, Rep. Math. Phys. 13 (1978) 149

3. H. Spohn, Rev. Mod. Phys. 53 (1980) 569

4. H.-P. Breuer and F. Petruccione, The Theory of Open Quantum Systems (Oxford University Press, Oxford, 2002)

5. F. Benatti and R. Floreanini, Int. J. Mod. Phys. B19 (2005) 3063

6. P. Zanardi, C. Zalka and L. Faoro, Phys. Rev. A 62 (2000) 030301; P. Zanardi, ibid. 63 (2001) 040304

7. J.I. Cirac, W. Dür, B. Kraus and M. Lewenstein, Phys. Rev. Lett. 86 (2001) 544

8. W. Dür, G. Vidal, J.I. Cirac, N. Linden and S. Popescu, Phys. Rev. Lett. 87 (2001) 137901

9. B. Kraus and J.I. Cirac, Phys. Rev. A 63 (2001) 062309

10. K. Życkowski, P. Horodecki, M. Horodecki and R. Horodecki, Phys. Rev. A 65 (2001) 012101

11. D. Braun, Phys. Rev. Lett. 89 (2002) 277901

12. M.S. Kim et al., Phys. Rev. A 65 (2002) 040101(R)

13. S. Schneider and G.J. Milburn, Phys. Rev. A 65 (2002) 042107

14. A.M. Basharov, J. Exp. Theor. Phys. 94 (2002) 1070

15. L. Jakobczyk, J. Phys. A 35 (2002) 6383

16. Z. Ficek and R. Tanas, Phys. Rep. 372 (2002) 369

17. B. Reznik, Found. Phys. 33 (2003) 167

18. F. Benatti, R. Floreanini and M. Piani, Phys. Rev. Lett. 91 (2003) 070402

19. F. Benatti and R. Floreanini, Phys. Rev. A 70 (2004) 012112

20. F. Benatti and R. Floreanini, J. Opt. B 7 (2005) S429

21. F. Benatti and R. Floreanini, Asymptotic entanglement of two independent systems in a common bath, Int. J. Quant. Inf., 2005, to appear

22. J.-H. An, S.-J. Wang and H.-G. Luo, J. Phys. A 38 (2005) 3579 
23. A.S. Holevo, Probabilistic and Statistical Aspects of Quantum Theory, (North Holland, Amsterdam, 1982)

24. G. Lindblad, J. Phys. A 33 (2000) 5059

25. J. Eisert and M.B. Plenio, Int. J. Quant. Inf. 1 (2003) 479

26. B.-G. Englert and K. Wódkiewicz, Int. J. Quant. Inf. 1 (2003) 153

27. A. Serafini, M. Paris, F. Illuminati and S. De Siena, J. Opt. B 7 (2005) R19

28. A. Ferraro, S. Olivares, M. Paris, Gaussian States in Continuous Variable Quantum Information, (Bibliopolis, Napoli, 2005)

29. S.L. Braunstein and P. van Loock, Rev. Mod. Phys. 77 (2005) 513

30. B. Demoen, P. Vanheuverzwijn and A. Verbeure, Rep. Math. Phys. 15 (1979) 27

31. P. Vanheuverzwijn, Ann. Inst. H. Poncaré A29 (1978) 123

32. F.A. Berezin, The Method of Second Quantization, (Academic Press, Orlando, 1966)

33. L.D. Faddeev, Introduction to functional methods, in Methods in Field Theory, R. Balian, J. Zinn-Justin, eds., (North-Holland, Amsterdam, 1976)

34. J.-P. Blaizot and G. Ripka, Quantum Theory of Finite Systems, (The MIT Press, Cambridge, 1986)

35. A. Perelomov, Generalized Coherent States and Their Applications, (Springer-Verlag, Berlin, 1986)

36. R. Simon, E.C.G. Sudarshan and N. Makunda, Phys. Rev. A 36 (1987) 3868

37. R. Simon, N. Makunda and B. Dutta, Phys. Rev. A 49 (1994) 1567

38. Arvind, B. Dutta, N. Makunda and R. Simon, Pramana, 45 (1995) 471

39. V. Gorini, A. Kossakowski and E.C.G. Sudarshan, J. Math. Phys. 17 (1976), 821;

40. G. Lindblad, Comm. Math. Phys. 48 (1976) 119

41. G. Lindblad, Rep. Math. Phys. 10 (1976) 393

42. A. Peres, Phys. Rev. Lett. 77 (1996) 1413

43. M. Horodecki, P. Horodecki and R. Horodecki, Phys. Lett. A 223 (1996) 1

44. R. Simon, Phys. Rev. Lett. 84 (2000) 2726

45. F. Benatti and R. Floreanini, J. Phys. A 33 (2000) 8139 